\title{
RESIDUOS AGROINDUSTRIALES COMO ADICIONES EN LA ELABORACIÓN DE BLOQUES DE CONCRETO NO ESTRUCTURAL
}

\section{AGRO-INDUSTRIAL WASTE AS ADDITIONS IN DEVELOPMENT OF CONCRETE BLOCKS NO STRUCTURAL}

\author{
Natalia Fuentes Molina', Oscar Iván Fragozo Tarifa², Lissette Vizcaino Mendoza³
}

Fecha de recepción: 17 de marzo de 2015

Fecha de aprobación: 4 de septiembre de 2015

Referencia: N. Fuentes Molina, O.I. Fragozo Tarifa, L. Vizcaino Mendoza. (2015). Residuos agroindustriales como adiciones en la elaboración de bloques de concreto no estructural. Ciencia e Ingeniería Neogranadina, 25(2), pp. 99 - 116, DOl: http://dx.doi.org/10.18359/rcin.1434

\section{RESUMEN}

La introducción de residuos industriales como sustitutos del cemento en bloques ecológicos de construcción, brindan la posibilidad de establecer un amplio desarrollo a nivel ambiental, social y económico. En la presente investigación se fabricaron bloques ecológicos con dimensiones comerciales a nivel industrial, en los que se reemplazó un porcentaje del contenido cemento por cascarilla de arroz, ceniza de la cascarilla de arroz y ceniza volante (caracterizadas mediante ensayos de granulometría, masa unitaria y humedad), en 10, 15 y $20 \%$, manteniendo constante la cantidad de agua y arena de mezclado del bloque. Los bloques ecológicos obtenidos se analizaron mecánicamente, y se determinó la resistencia a compresión, obteniendo como resultados promedio $0.585 \mathrm{MPa}, 0,743 \mathrm{MPa}$ y 0,956 MPa para cascarilla de arroz, ceniza de la cascarilla de arroz y ceniza volante, respectivamente, a los 7, 28 y 45 días de curado; dichas resistencias se compararon con la del patrón, que consistían en el bloque de referencia con 100\% de cemento, las cuales fueron de $0.802 \mathrm{MPa}$, para observar las características cementantes de las adiciones, las cuales afectan considerablemente la resistencia del eco-bloque. Mediante los resultados obtenidos, se concluyó que el porcentaje óptimo de adición es el $15 \%$ de ceniza de centrales térmicas a los 28 días de curado, como reemplazo parcial del cemento en bloques de concreto, resaltando que aunque existe una disminución en la resistencia a la compresión

\footnotetext{
1. Magister en Ciencias Ambientales, Grupo Madre Tierra, Universidad de La Guajira, Facultad de Ingeniería, nnfuentes@uniguajira.edu.co

2. Ing. Minas, Universidad de La Guajira, osfragozo@yahoo.com

3. Ing. Medio Ambiente Grupo Madre Tierra, Universidad de La Guajira.
} 
y tensión en algunos casos, es viable el uso de las adiciones para la elaboración de bloques de concreto como propuesta efectiva para la reutilización de estos residuos que sirven de guía hacia el desarrollo de materiales más competitivos técnica, económica y ambientalmente.

Palabras clave: Cenizas Industriales, Residuo Agrícola, Aditivo Residual, Eco-bloques.

\section{ABSTRACT}

The introduction of industrial waste as a substitute for cement in organic building blocks makes it possible a broad environmental, social and economic development. In this research ecological blocks were made with commercial dimensions at industrial level, in which a percentage of the cement content was replaced with rice husk, rice husk ashes and fly ash (characterized by tests of particle size, unit weight and moisture ) in 10, 15 and $20 \%$; keeping the amount of mixing water and sand block constant. The obtained organic blocks were mechanically analyzed, and the compressive strength was determined, obtaining average results of $0.585 \mathrm{MPa}, 0.743 \mathrm{MPa}$ and $0.956 \mathrm{MPa}$ for rice husk, rice husk ash and fly ash respectively at 7,28 and 45 days of curing. Those resistances were compared with the reference $100 \%$ cement block, resulting in 0.802 $\mathrm{MPa}$, in order to observe cementitious characteristics of the additions, which greatly affect the eco-block resistance. Through the results obtained, it was concluded that the optimum addition rate is $15 \%$ of ashes from thermal power plants at 28 days of curing, as a partial replacement of cement in concrete blocks. Although there is a decrease in resistance compression and tension in some cases, it is feasible using additions in the making of concrete blocks as an effective proposal for reusing those wastes and lead towards the development of technical, economic and environmentally competitive materials.

Keywords: Industrial Ashes, Agricultural Waste, Residual Additive, Eco-blocks.

\section{INTRODUCCIÓN}

En la actualidad la investigación, la transferencia de tecnologías, los nuevos materiales de ingeniería y la utilización de los residuos industriales generados tienen un papel importante para el desarrollo, al generar innovación y mejora que fortalecen la producción de productos y la prestación de servicios amigables con el medio ambiente [1]. La obtención de cemento para fabricar concreto implica un alto consumo energético y grandes emisiones de gases, lo que lo convierte en un material costoso y contaminante. La industria del cemento, a escala mundial, tiene el $2 \%$ del consumo global de energía y el $5 \%$ del consumo global de energía industrial [2], lo que genera aproximadamente una tonelada de $\mathrm{CO}_{2}$ por cada tonelada de clinker dependiendo de la eficiencia de la planta [3]. En algunos casos se podría sustituir el cemento por adiciones de residuos agroindustriales, con muy buenos resultados [4-5].

El empleo de estas adiciones como sustituto de cierto porcentaje de cemento disminuye de 
forma significativa el costo medioambiental de la construcción, al reducir parte de la generación de $\mathrm{CO}_{2}$ y explotación minera necesarias para la producción de cemento, además de mejorar la gestión de los residuos reutilizados y evitar su disposición en botaderos [6].

En la presente investigación se evaluó el aprovechamiento de la cascarilla de arroz, las ceniza de la cascarilla de arroz y las ceniza de las centrales térmicas como adiciones residuales en bloques ecológicos de mampostería dadas las ventajas que ofrecen, permitiéndonos avanzar en el conocimiento de las características físicas, químicas y mecánicas de las adiciones.

La cascarilla de arroz representa, aproximadamente, una quinta parte en peso del fruto recolectado, variando esta cantidad en función de las condiciones de cultivo y la variedad del arroz [7-8]; es, a su vez, un desecho agroindustrial que constituye aproximadamente el $20 \%$ de la producción mundial de arroz, con estimaciones cercanas a los $7 \times 10^{8}$ toneladas anuales [9], siendo uno de los mayores residuos resultante de la producción agrícola de los países productores de arroz.

En Colombia, la producción de arroz está cercana a 2.1×106 toneladas/año, y como consecuencia de esta producción cerca de $4 \times 10^{5}$ toneladas de cascarilla de arroz como residuo, las cuales mediante un proceso de quema controlada dan origen a cerca de $1 \times 10^{5}$ toneladas de cenizas con un alto contenido de sílice [9], la alta disponibilidad de estos residuos y sus características los convierten en una alternativa efectiva para la industria de la construcción [10-13].

En la actualidad Colombia produce más de $8 \times 10^{7}$ toneladas de carbón para abastecer el sector doméstico e industrial [14], se considera que anualmente se queman más de $8.3 \times 108$ toneladas de carbón que generan unos $7.5 \times 10^{7}$ toneladas de residuos de combustión (0.8 ton de cenizas volantes/ton de carbón) formados principalmente por cenizas volantes [15]; la alta producción de cenizas de centrales térmicas en Colombia hace viable la intención de aprovechamiento de estos residuos en la industria de la construcción como sustituto del cemento [16-18].

Finalmente, se plantea que existe una gran problemática social, económica y ambiental, que requiere introducir estrategias que promuevan su solución o mitigación y así reducir los altos impactos ambientales que se evidencian. ¿Cuál sería el porcentaje óptimo de adición residual en la elaboración de bloques de construcción como opción sostenible para las comunidades vulnerables?

\section{MATERIALES Y MÉTODOS}

La evaluación del uso de los residuos agroindustriales como adiciones en la elaboración de bloques de construcción, se realiza en tres momentos i. Recolección y caracterización de los residuos agroindustriales procedentes de arroceras y centrales térmicas con los ensayos físicoquímicos de los materiales residuales ii. Selección de los porcentajes de las adiciones y los días de curado para la construcción de los bloques de concreto, y iii. Determinación de las características mecánicas de los bloques, para así establecer la viabilidad de su uso a partir de la normativa y las diferencias de los bloques de construcción con adición residual y los bloques de construcción comerciales. 


\subsection{RECOLECCIÓN Y CARACTERIZACIÓN DE LOS RESIDUOS AGROINDUSTRIALES}

Se seleccionaron los residuos agroindustriales cascarilla de arroz, las ceniza de la cascarilla de arroz y las cenizas de las centrales térmicas como adiciones en la elaboración de los bloques de concreto mediante el análisis de referencias que emplearon diferentes adiciones residuales, permitiéndonos realizar la selección de los mismos, según la disponibilidad y los problemas ambientales por la disposición inadecuada; como se observa en la tabla 1.

Tabla 1. Tipos de adiciones residuales evaluados en la elaboración de bloques de construcción en diferentes regiones.

\begin{tabular}{|c|c|c|}
\hline Adiciones residuales & Lugar & Referencias \\
\hline Cascarilla de arroz & Guatemala, España, Argentina, Perú, Colombia & {$[18-23]$} \\
\hline Cenizas de cáscara de arroz & $\begin{array}{c}\text { España; Perú; Argentina; Habana; Cuba; } \\
\text { Cartagena; Colombia. }\end{array}$ & {$[1,24-26]$} \\
\hline Ceniza centrales térmicas & $\begin{array}{c}\text { Cali; Colombia; España; Argentina; Medellín; } \\
\text { Colombia Cali; Colombia }\end{array}$ & {$[27-35]$} \\
\hline Arena & Perú & {$[36]$} \\
\hline Ceniza de rastrojo de maíz & España & {$[37]$} \\
\hline Ceniza volante y piedra pómez & Bucaramanga; Colombia & {$[21)$} \\
\hline Cenizas hoja de maíz y bagazo de caña & Venezuela & {$[23]$} \\
\hline Ceniza de coco y pino & Inglaterra & {$[34]$} \\
\hline
\end{tabular}

Una vez realizada la selección de las adiciones, en reemplazo del cemento, se realiza la caracterización de los materiales, mediante los ensayos de densidad, masa unitaria (compactada y suelta), absorción y granulometría, y contenido de humedad; dióxido de silicio $\mathrm{SiO}_{2}$ óxido de aluminio $\mathrm{Al}_{2} \mathrm{O}_{3^{\prime}}$ óxido de hierro $\mathrm{Fe}_{2} \mathrm{O}_{3}$ y trióxido de azufre $\mathrm{SO}_{3}$ presentados en la tabla 2 .

Cemento: para la elaboración de los bloques de concreto el cemento utilizado fue el Portland gris tipo I comercializado en el país, el cual, de acuerdo con el fabricante, cumple las especificaciones físicas, mecánicas NTC 121 y químicas NTC 321.
Cenizas centrales térmicas: las cenizas de centrales térmicas es el residuo de combustión de carbón en el proceso de generación de energía de la planta Gecelca, ubicada en el municipio de Riohacha, La Guajira, Colombia, que sale con los gases de la cámara de combustión por ductos de conducción mediante filtros electrostáticos, también llamadas cenizas volantes.

Cenizas de cascarilla de arroz: las cenizas de cascarilla de arroz empleadas provienen de la arrocera La Gloria, ubicada en el municipio Fonseca, La Guajira, Colombia, después de un proceso de combustión controlado. 
Tabla 2. Caracterización fisicoquímica de los materiales residuales utilizados en la elaboración de bloques de construcción

\begin{tabular}{|c|c|c|c|c|}
\hline CARACTERÍstICAS & NORMAS & $\begin{array}{c}\text { CENIZAS } \\
\text { CENTRALES } \\
\text { TÉRMICAS }\end{array}$ & $\begin{array}{c}\text { CENIZAS } \\
\text { CASCARILA } \\
\text { DE ARROZ }\end{array}$ & $\begin{array}{c}\text { CASCARILLA } \\
\text { DE ARROZ }\end{array}$ \\
\hline Contenido de Humedad & NTC 3493 & 0.316 & 0.852 & 21,3 \\
\hline Densidad $\left(\mathrm{g} / \mathrm{cm}^{3}\right)$ & NTC 221 & 2.21 & 2.98 & 7.84 \\
\hline Masa unitaria suelta $\left(\mathrm{g} / \mathrm{cm}^{3}\right)$ & INV.E 217-07 NTC 92 & 0.623 & 0.350 & 0.128 \\
\hline Masa unitaria compacta $\left(\mathrm{g} / \mathrm{cm}^{3}\right)$ & INV.E 217-07 NTC 92 & 0.693 & 0.417 & 0.188 \\
\hline Absorción & INV.E 222-07 NTC 176 & 2.93 & 5.18 & 4.44 \\
\hline Dióxido de silicio $\mathrm{SiO}_{2^{\prime}}$ & NTC 3493 & 42.11 & 90.89 & 84.6 \\
\hline Óxido de aluminio $\mathrm{Al}_{2} \mathrm{O}_{3}$ & NTC 3493 & 30.26 & 0.21 & 0.92 \\
\hline Óxido de hierro $\mathrm{Fe}_{2} \mathrm{O}_{3}$ & NTC 3493 & 4.70 & 0.47 & 0.47 \\
\hline Trióxido de azufre $\mathrm{SO}_{3}$ & NTC 3493 & 0.01 & 0.03 & 1.03 \\
\hline
\end{tabular}

Cascarilla de arroz: la cascarilla de arroz empleada proviene de la arrocera La Gloria, ubicada en el municipio Fonseca, La Guajira, Colombia; a esta se le realizó un lavado con el fin de eliminar las impurezas (materiales propios de los procesos a los que se ve sometido) principalmente material fino (polvillo), restos de la paja y granos de arroz.

Arena: esta fue de origen natural, libre de materiales contaminantes e impurezas orgánicas, bien dragada (partículas de todos los tamaños), lo que garantizó una mezcla trabajable y adherente que cumplió con las especificaciones en NTC 2240 en sus características granulométricas, $0.350 \mathrm{~g} /$ $\mathrm{cm}^{3}$ peso unitario suelto y $0.417 \mathrm{~g} / \mathrm{cm}^{3}$ peso unitario compacto.

Las características de la distribución granulométricas en la tabla 3 de los materiales residuales condicionan las relaciones en forma diferente, cemento: cenizas centrales térmicas, cemento: cenizas cascarilla de arroz y cemento: cascarilla de arroz, formando zonas topoquímicas de mayor reactividad cementicia en granos menores que en granos mayores, como fue evidenciado en los resultados obtenidos.

\subsection{SELECCIÓN DE LOS PORCENTAJES DE LAS ADICIONES Y LOS DÍAS DE CURADO PARA LA CONSTRUCCIÓN DE LOS BLOQUES DE CONCRETO}

Se elaboraron un total de 108 eco-bloques de construcción para las pruebas de resistencia a compresióny tensión, con diferentes porcentajes de residuos agroindustriales (cascarilla de arroz, las cenizas de la cascarilla de arroz y las cenizas de la centrales térmicas), establecidos mediante el análisis de varias investigaciones (donde se elaboraron bloques ecológicos de concreto) presentados en la tabla 4; seleccionando así 
Tabla 3. Caracterización granulométrica de los materiales residuales utilizados en la elaboración de bloques de construcción.

\begin{tabular}{|c|c|c|c|c|c|c|}
\hline \multicolumn{7}{|c|}{ Análisis granulométrico de los materiales residuales NTC 77 } \\
\hline Tamiz (mm) & $\mathbf{4 . 7 6}$ & $\mathbf{2 . 3 8}$ & $\mathbf{2 . 0}$ & $\mathbf{1 . 1 9}$ & $\mathbf{0 . 4 2 5}$ & $\mathbf{0 . 0 7 5}$ \\
\hline $\begin{array}{c}\text { \% Que pasa de Cenizas } \\
\text { centrales térmicas }\end{array}$ & 100 & 100 & 100 & 100 & 90.2 & 37.9 \\
\hline $\begin{array}{c}\text { \% Que pasa de Cenizas } \\
\text { cascarilla de arroz }\end{array}$ & 100 & 100 & 100 & 100 & 83.9 & 29.8 \\
\hline \% Que pasa cascarilla de arroz & 100 & 100 & 98.4 & 73.2 & 28.1 & 11.4 \\
\hline$\%$ Que pasa Arena & 100 & 97.8 & 83.7 & 41.6 & 25.9 & 1.6 \\
\hline
\end{tabular}

Tabla 4. Porcentajes de adición residuales evaluados en la elaboración de bloques de construcción en diferentes regiones.

\begin{tabular}{|c|c|c|}
\hline ADICIÓNES RESIDUALES & PORCENTAJES & REFERENCIAS \\
\hline Cascarilla de arroz & $0,4-80$ & {$[19-22,24]$} \\
\hline Cenizas centrales térmicas & $0,4-33$ & {$[29-34]$} \\
\hline Ceniza de la cascarilla de arroz & $5-45$ & {$[1,24-26]$} \\
\hline Cenizas y escorias de alto horno & $15-40$ & {$[27]$} \\
\hline Arena & 70 & {$[36]$} \\
\hline ceniza de rastrojo de maíz & 10 & {$[37]$} \\
\hline Las cenizas de hoja de maíz y de bagazo de caña & $10-30$ & {$[34]$} \\
\hline Ceniza de coco y pino & 20 & \\
\hline
\end{tabular}

para esta investigación porcentajes del 10, 15 y $20 \%$ de adición de material residual.

Además, se elaboraron 12 bloques con $100 \%$ de cemento para los diferentes días de curado, siendo este el bloque patrón; es decir, los bloques con los que se comparó la resistencia de los eco-bloques. En este caso los bloques y eco-bloques diseñados son de tipo comercial de doble hueco; con dimensiones de $8,7 \mathrm{~cm}$ de ancho, 15,8 cm de alto, $35 \mathrm{~cm}$ de largo y
6,3 kg de peso neto, como se muestra en la figura 1.

Los bloques fueron curados durante 7, 28 y 45 días para hacer los análisis correspondientes de resistencia a compresión y tensión; durante estos días se remojaron los bloques 3 veces diarias para aumentar su resistencia. Estos días fueron establecidos tomando como referencia los de curado evaluados en las investigaciones presentadas en la tabla 5 en 

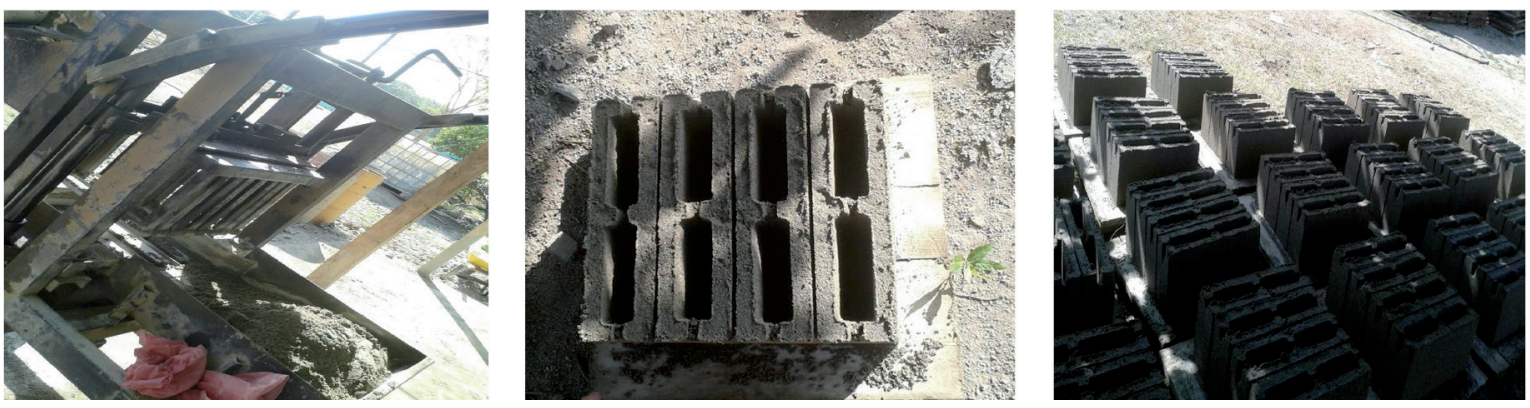

Figura 1. Elaboración de bloques de construcción con adición de cenizas centrales térmicas; cascarilla de arroz y cenizas cascarilla de arroz.

Tabla 5. Días de curado evaluados en diferentes adición residuales evaluados en la elaboración de bloques de construcción en diferentes regiones.

\begin{tabular}{|c|c|c|}
\hline ADICIÓNES RESIDUALES & DÍAS DE CURADO & REFERENCIAS \\
\hline Cascarilla de arroz & $1-28$ & {$[19,20-21,24]$} \\
\hline Cenizas centrales térmicas & $1-90$ & {$[22,31-34]$} \\
\hline Ceniza de la cascarilla de arroz & $7-91$ & {$[1,23-27]$} \\
\hline Cenizas y Escorias de alto horno. & 28 & {$[27]$} \\
\hline Ceniza de rastrojo de maíz & $28-90$ & {$[36]$} \\
\hline Ceniza volante y piedra pómez. & $7-28$ & {$[21]$} \\
\hline Cenizas de hoja de maíz y bagazo de caña & $7-90$ & {$[23]$} \\
\hline Ceniza de coco y pino & $7-28$ & {$[35]$} \\
\hline
\end{tabular}

bloques ecológicos con diferentes adiciones residuales.

Para la fabricación de todos los bloques de construcción con adición de los materiales residuales (cenizas centrales térmicas, cenizas cascarilla de arroz y cascarilla de arroz) y sin adición, se tomaron en cuenta las proporciones calculadas por bloques a nivel volumétrico con el objetivo de conocer las mejores adiciones y porcentajes. En la mezcla destinada a la elaboración de los bloques se empleó arena con partículas de diversos tamaños; agua, la cual fue suministrada mediante dos aplicaciones diferentes, la primera hidratando la mezcla y la segunda manteniendo el aire saturado de agua, tratando de regularlo para producir mejor trabajabilidad, asentamiento y fluidez que facilitara el trabajo en la obtención de los bloques, como se aprecia en la figura 1.

En la elaboración de los bloques se utilizó una máquina ponedora manual sólida y de fácil manejo, con capacidad para 4 bloques, con un proceso de prensado hidráulico que compacta una mezcla de arena; material residual, agua 
y cemento [23]. La prensa está fabricada completamente de acero, tiene una caja molde en la cual un pistón operado a mano comprime la mezcla ligeramente húmeda. Una vez que el bloque es compactado, se apilan en grupo de cuatro unidades en una tabla previamente colocada en la máquina, ya que los bloques deben salir de esta puestos en tablones lisos, lo suficientemente anchos para ser curados las 24 horas del día [36].

\subsection{DETERMINACIÓN DE LAS CARACTERÍSTICAS MECÁNICAS DE LOS BLOQUES DE CONCRETO}

Terminado el proceso de curado de los bloques elaborados se realizan los análisis de resistencia a compresión y tensión para determinar la calidad de los elaborados en cada una de las combinaciones realizadas según la norma NTC 4076 y ASTM C129, donde se establece el procedimiento de las unidades bloques y ladrillos de concreto no estructural, y nos permitió evaluar la calidad del producto elaborado.

Para la realización de este ensayo se fracturaron los bloques con prensas italianas automáticas de última generación (Automax 5; Control S) que ejerce una carga continua sobre los bloques a una velocidad tal que permite que la carga máxima se alcance en no menos de 20 segundos y no más de 80 segundos, hasta completar la rotura de la misma. Para los ensayos de resistencia a la compresión se colocó la probeta lo más centrada posible entre los dos platos de la máquina de compresión [22]. Los bloques ecológicos y comerciales soportaron fuerzas desde $580.145 \mathrm{kgf}$ hasta $3691.335 \mathrm{kgf}$, obteniendo así las resistencias a la compresión. Para los ensayos de resistencia a la tensión se necesitó anexarle a la máquina un par de placas metálicas, donde la carga se aplicó con el rodillo superior sin aceleraciones bruscas y a una velocidad tal, de modo que la duración del ensayo estuvo comprendida entre los 30 y 90 segundos $[22,38]$, soportando fuerzas desde 259 kgf hasta 700 kgf, obteniendo así el registro de la carga máxima soportada, la cual es relacionada con la sección transversal de los bloques.

\section{RESULTADOS Y DISCUSIÓN}

A continuación se presentan, en la tabla 6 y figura 2, los resultados de los diferentes ensayos realizados con los tres porcentajes de adición: 10, 15 y $20 \%$; por cada adición residual (cenizas centrales térmicas; cascarilla de arroz y cenizas cascarilla de arroz) empleada como adición del aglomerante en sus días de curado.

\subsection{ANÁLISIS DE LA RESISTENCIA A LA COMPRESIÓN POR ADICIÓN EN LOS DIFERENTES DÍAS DE CURADO}

Al observar la resistencia a compresión de los bloques ecológicos con cenizas de cascarilla de arroz, se evidencia que la resistencia es inversamente proporcional al porcentaje, teniendo en cuenta que al aumentar la cantidad de adición disminuye la resistencia. La resistencia de los bloques ecológicos con el $10 \%$ de adición es superior a la presentada por el bloque comercial; los del $15 \%$ son valores similares al bloque comercial, y los del $20 \%$ son inferiores. La mayor resistencia se registra en el eco-bloque, con porcentajes del $10 \%$ a los 28 días.

Los bloques elaborados con cascarilla de arroz, con porcentajes del 15 y del $20 \%$ a los 7, 28 y 45 días, en su mayoría presentan menor resistencia a la compresión en comparación 
Tabla 6. Resultados obtenidos de los análisis mecánicos resistencia a la compresión y tensión de los bloques ecológicos y comerciales para diferentes porcentajes y días de curado.

\begin{tabular}{|c|c|c|c|c|c|c|c|}
\hline \multirow{3}{*}{ Adiciónes residuales } & \multirow{3}{*}{$\begin{array}{l}\text { Porcentajes } \\
\text { (\%) }\end{array}$} & \multicolumn{6}{|c|}{ Análisis Mecánicos } \\
\hline & & \multicolumn{3}{|c|}{ Compresiōn (MPa) } & \multicolumn{3}{|c|}{ Tensión (MPa) } \\
\hline & & 7 dias & 28 días & 45 días & 7 dias & 28 días & 45 días \\
\hline \multirow{3}{*}{ Cenizas cáscara de arroz } & 10 & 1,022 & 1,439 & 0,435 & 0,061 & 0,079 & 0,054 \\
\hline & 15 & 0,822 & 0,943 & 0,397 & 0,052 & 0,076 & 0,072 \\
\hline & 20 & 0,611 & 0,502 & 0,515 & 0,044 & 0,052 & 0,058 \\
\hline \multirow{3}{*}{ Cenizas centrales térmicas } & 10 & 1,340 & 1,147 & 0,635 & 0,058 & 0,100 & 0,078 \\
\hline & 15 & 0,899 & 1,235 & 0,823 & 0,043 & 0,097 & 0,073 \\
\hline & 20 & 0,688 & 1,162 & 0,680 & 0,071 & 0,121 & 0,079 \\
\hline \multirow{3}{*}{ Cáscara de arroz } & 10 & 1,084 & 1,040 & 0,573 & 0,059 & 0,086 & 0,063 \\
\hline & 15 & 0,355 & 0,518 & 0,403 & 0,036 & 0,062 & 0,058 \\
\hline & 20 & 0,446 & 0,438 & 0,358 & 0,052 & 0,057 & 0,048 \\
\hline Patrón & - & 0,815 & 1,046 & 0,546 & 0,074 & 0,074 & 0,067 \\
\hline
\end{tabular}

con el patrón; excepto los bloques con el $10 \%$ de adición a los 7 y 45 días, con 1.084 y 0.573 $\mathrm{MPa}$, que obtuvieron una mayor resistencia, siendo similares al patrón. Esta tendencia puede ser explicada por la baja actividad de la cascarilla de arroz en cuanto a su estructura cristalina y al tamaño de la partícula.

Con respecto a las cenizas de las centrales térmicas, se observó que la resistencia disminuye a medida que incrementan los días de curado; excepto para los bloques ecológicos con el $20 \%$ de la adición residual; pero se encontró que todos los valores de resistencia están por encima de los obtenidos por el bloque comercial; excepto para los bloques ecológicos con el porcentaje del $20 \%$ a los 7 días. Y la mayor resistencia se obtuvo a los 7 y 28 días de curado con el $10 \%$ de adición. En términos generales, se encontró que los bloques elaborados con cenizas centrales térmicas presentan mayores resistencias a la compresión en comparación con el bloque comercial y los bloques con cenizas arroceras y cáscara de arroz, la razón por la cual se presenta esta tendencia seguramente es por el tamaño de las partículas del material (cenizas térmicas) que al ser más finas que los otros materiales facilitan la activación química de la mezcla e incrementan la resistencia a la compresión.

En relación con el porcentaje manejado para los bloques ecológicos, siguen siendo más representativos los bloques con el $10 \%$ de las adiciones residuales para las tres adiciones cenizas arroceras; de cáscara de arroz y cenizas centrales térmicas, con valores de $1.439,1,340$ y $1,084 \mathrm{MPa}$; esto posiblemente es debido a que son las relaciones más bajas de cemento/material residual, lo cual tiene mayor cantidad de cemento por unidad de volumen del bloque estructurado, que aumenta la 

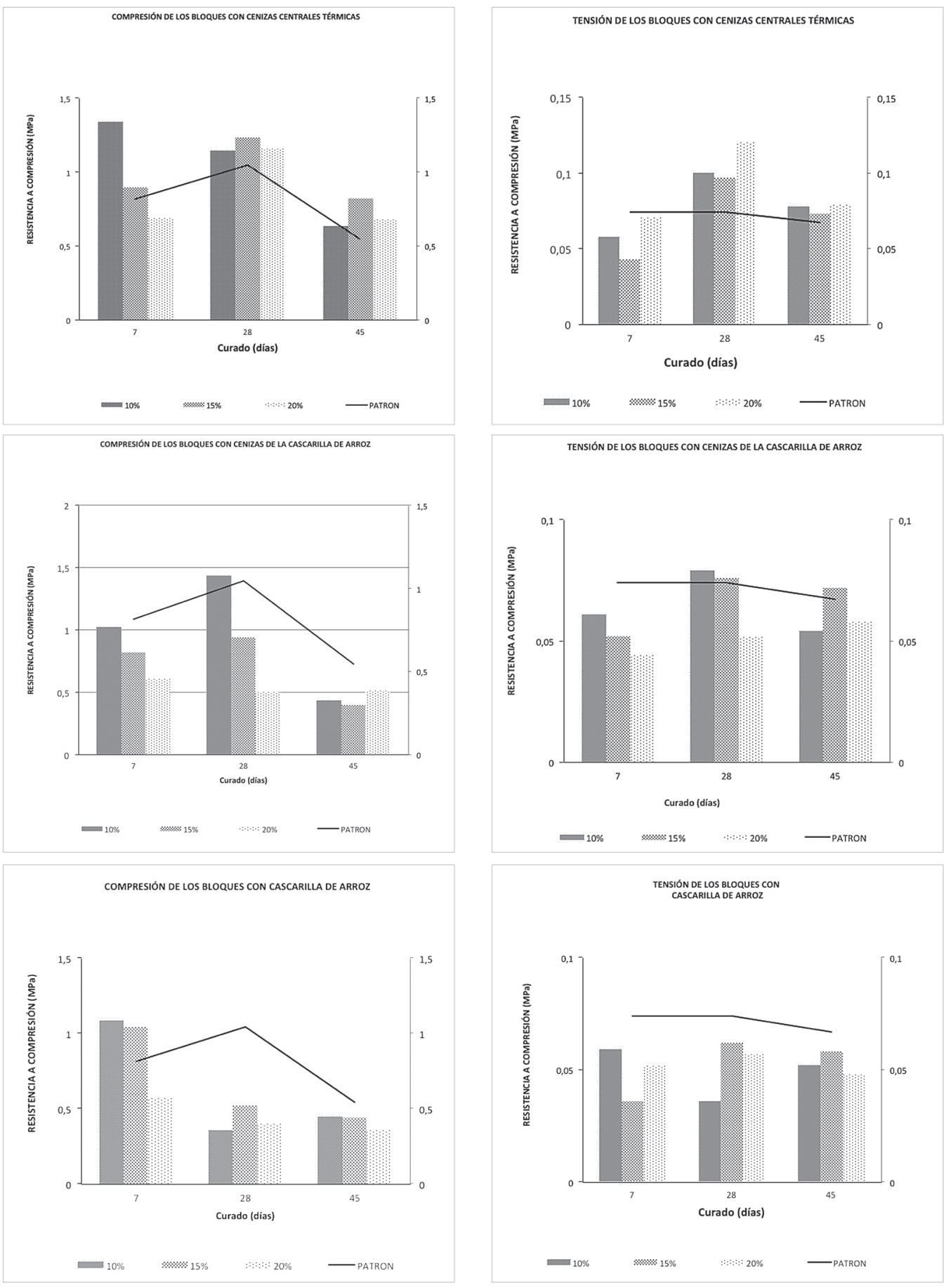

Figura 2. Resistencia a la compresión (MPa) y resistencia a la tensión (MPa) para bloques comerciales y bloques ecológicos en los diferentes días de curado. Donde las barras representan los bloques ecológicos y la curva representa

el bloque comercial. 
capacidad cementante en relación con las demás proporciones.

Los bloques elaborados con adiciones de material residual nocumplen con losestándares mínimos de mampostería estructural NTC 4026 y mampostería no estructural NTC 4076 de bloques de concreto, por estar por debajo del rango de valores de resistencia de 5.49 a 6.08 MPa, por lo que es recomendable realizar un pre-tratamiento a los materiales residuales previo a la mezcla de concreto para aumentar la reactividad química que contribuyan a incrementar la resistencia.

Al comparar los resultados obtenidos en los análisis mecánicos de resistencia a la compresión para los bloques ecológicos de la presente investigación con otros autores presentados en la tabla 7, se encuentra que la cascarilla de arroz presenta valores similares en los plasmados por [20] y [22]. En comparación con las cenizas arroceras, se encontraron valores inferiores a los de [1, 24, 26, 39]; igual sucede en el caso de las cenizas centrales térmicas, para las cuales se encontraron valores inferiores a los de [29-32]. Razón por la cual no es posible establecer un patrón del comportamiento de la resistencia a la compresión de las adiciones trabajadas, debido a que las mayores resistencias se presentan con adiciones (variabilidad asociada con las microestructuras amorfas y los tamaños de partículas) y porcentajes diferentes.

Como se puede observar, los resultados promedio para cascarilla de arroz (0.585 MPa), cenizas cascarilla de arroz ( $0.743 \mathrm{MPa}$ ) y cenizas volante (0.956 MPa) no cumplen con la Norma NTC 4076 para la elaboración de bloques no estructurales, lo que demuestra que es inviable la incorporación de cantidades de residuos agroindustriales, porque estas requieren de grandes volúmenes de agua, lo que ocasiona la segregación de los materiales y el cemento adicionado, e imposibilita el fraguado de los bloques, como se evidenció en la presente investigación. Se recomienda la utilización de un mezclado más controlado para mejorar la calidad de la mezcla, creando una zona de interfase más resistente para reforzar las áreas débiles al rellenar los poros y grietas de los bloques de concreto, como lo menciona [10].

\subsection{ANÁLISIS DE LA RESISTENCIA A LA TENSIÓN POR ADICIÓN ORGÁNICA EN LOS DIFERENTES DÍAS DE CURADO}

Los análisis de resistencia a la tensión realizados a los bloques de construcción con cada porcentaje de cenizas de cascarilla dearroz y sus respectivos días de curado, muestran que la resistencia a la tensión con el 10 y $15 \%$ es similar en comparación con el bloque comercial; mientras los del $20 \%$ presentaron valores inferiores. A su vez, los bloques con el 10 y el $15 \%$ de esta adición presentaron la mayor resistencia a los 28 días de curado. En términos generales, el comportamiento evidenciado en las relaciones porcentajes de cenizas y días de curado es directamente proporcional, lo cual era de esperarse por la humedad que confieren estos materiales al bloque elaborado.

Los bloques elaborados con cascarilla de arroz con el 10 y el $20 \%$ presentan valores similares al bloque comercial; mientras que aquellos que contienen el $15 \%$ obtuvieron valores inferiores de resistencia a la tensión; las mayores resistencias a la tensión fueron los del $10 \%$ a los 28 días de curado. De igual forma, los bloques de ceniza de las centrales térmicas a los 28 días de curado son los que obtuvieron mayor resistencia en comparación 
Tabla 7. Resistencia a la compresión de adiciones residuales evaluados en la elaboración de bloques de construcción en diferentes autores.

\begin{tabular}{|c|c|c|}
\hline \multirow{2}{*}{ ADICIÓN RESIDUAL } & RESISTENCIA & \multirow{2}{*}{ REFERENCIAS } \\
\hline & COMPRESIÓN PSI & \\
\hline Cascarilla de arroz & $0.380-13.300$ & [19] \\
\hline Cenizas centrales térmicas & $7.356-11.964$ & [29] \\
\hline Ceniza volante & $4.021-10.004$ & {$[30]$} \\
\hline cenizas de cáscara de arroz y cascarillas de arroz & $2.999-15.000$ & {$[24]$} \\
\hline Cascarilla de arroz & $0.430-13.400$ & {$[20]$} \\
\hline Cenizas de la cascarilla de arroz & $257.001-416.001$ & {$[1]$} \\
\hline cenizas de cáscara de arroz & $9.400-46.500$ & {$[26]$} \\
\hline Cenizas centrales térmicas ; & $26.660-60.030$ & [19] \\
\hline Ceniza de rastrojo de maíz & $39.860-61.350$ & {$[37]$} \\
\hline Ceniza volante y piedra pómez. & $0.589-31.302$ & {$[21]$} \\
\hline Ceniza volante & $8.045-12.810$ & {$[31]$} \\
\hline ceniza volante & $29.424-34.916$ & {$[32]$} \\
\hline Cenizas de la cáscara de arroz & $20.560-57.030$ & [39] \\
\hline Cenizas centrales térmicas & $20.000-35.000$ & [33] \\
\hline Cenizas de hoja de maíz; de cascarilla de arroz y de bagazo de caña & $6.473-53.454$ & [23] \\
\hline Ceniza volante & $16.140-31.910$ & [34] \\
\hline Ceniza de coco y pino & $10.342-55.158$ & [35] \\
\hline Cenizas centrales térmicas y cascarilla de arroz & $0.695-24.570$ & {$[21,35,40]$} \\
\hline
\end{tabular}

con los bloques comerciales, específicamente los porcentajes del 10 y $20 \%$.

Se encontró que a los 28 días se presentan para los bloques ecológicos elaborados con las tres adiciones: cenizas arroceras, cáscara de arroz y cenizas centrales térmicas, las mayores resistencias a la tensión, con valores de 1.135, 1.221 y $1.727 \mathrm{MPa}$, respectivamente, siendo este último el valor más alto; lo que implica que los bloques ecológicos con ceniza de las centrales térmicas resultan ser los más resistentes.

Se logra apreciar que a los 7 días de curado todas la adiciones presentan resistencias a la tensión similares, lo cual es debido a que apenas ha comenzado la relación puzolánica, dado que a los 28 días se evidencia un incremento notable generado por la mayor reacción puzolánica y diferencias entre las diferentes cenizas residuales evaluadas. 
En la mayoría de los casos también se encuentra que los porcentajes del $10 \%$ presentan alta resistencia a la tensión, indicándose que este representa el porcentaje óptimo, una vez comparado con el patrón comercial. Lo anterior indica que hay un aumento en la tensión conforme disminuye el porcentaje cemento material residual, lo cual podría ser atribuido a la homogeneización del material en el bloque.

En relación con la resistencia a la tensión para las adiciones orgánicas de cenizas arroceras y cascarilla de arroz empleados, la tabla 8 ilustra el comportamiento de estas variables en otras investigaciones, encontrando valores inferiores a [39] y similares a [34] y Gonzales [26], arrojando resultados de resistencia más altos para la adición orgánica de cenizas arroceras. Por ser diferencias pequeñas, pueden ser asociadas a las variabilidades propias de estos materiales, que bajo este principio presentarían reactividades diferentes.

\subsection{ANÁLISIS COMPARATIVO DEL COMPORTAMIENTO MECÁNICO DE LOS BLOQUES CON LAS ADICIONES DE MATERIAL RESIDUAL EN SUS DIFERENTES DÍAS DE CURADO}

Al fallar los bloques con las adiciones residuales, para los análisis de compresión, a los 7 días todos los bloques del $10 \%$ obtuvieron una mayor resistencia en comparación con el bloque comercial. A los 28 días los bloques con contenidos de CCA y CT resisten una mayor compresión en comparación con el bloque comercial, presentado en la figura 3.

Los ensayos realizados con la cáscara de arroz fueron inviables cuando los porcentajes fueron grandes, toda vez que se produjeron demandas de agua más altas en comparación con las demás adiciones, comportamiento que se atribuye a la morfología de la cáscara, situación que se puede solucionar al moler la cáscara previamente para mejorar las relaciones de cemento cascarilla de arroz [40]. Atribuye este comportamiento a la forma cóncava de la cáscara y a las densidades que están por debajo de $1,5 \mathrm{~g} / \mathrm{cm}^{3}$, lo cual imposibilita el fraguado, por lo que se sugiere utilizar tensoactivos para facilitar la mezcla de los componentes del bloque.

Se observa cómo la resistencia a la tensión de los bloques elaborados con el $10 \%$ de las diferentes adiciones cáscara de arroz, cenizas de cáscara de arroz y cenizas de centrales térmicas, con 7 y 45 días de curado es inferior en comparación con el bloque comercial similar al análisis de compresión, siendo los 28 días de curado el tiempo óptimo presentado en esta investigación.

Tabla 8. Resistencia a la tensión de adiciones residuales evaluadas en la elaboración de bloques de construcción en diferentes regiones.

\begin{tabular}{|c|c|c|}
\hline \multirow{2}{*}{ ADICIŌN RESIDUAL } & RESISTENCIA & \multirow{2}{*}{ REFERENCIAS } \\
\cline { 2 - 2 } & TENSIÓN MPa & \\
\hline Cascarilla de arroz & $0.343-1.951$ & {$[34]$} \\
\hline cenizas de cáscara de arroz & $1.285-6.766$ & {$[26]$} \\
\hline Cáscara de arroz & $1.373-2.844$ & {$[23]$} \\
Cenizas de la cáscara de arroz & $4.991-9.807$ & {$[39]$} \\
\hline
\end{tabular}



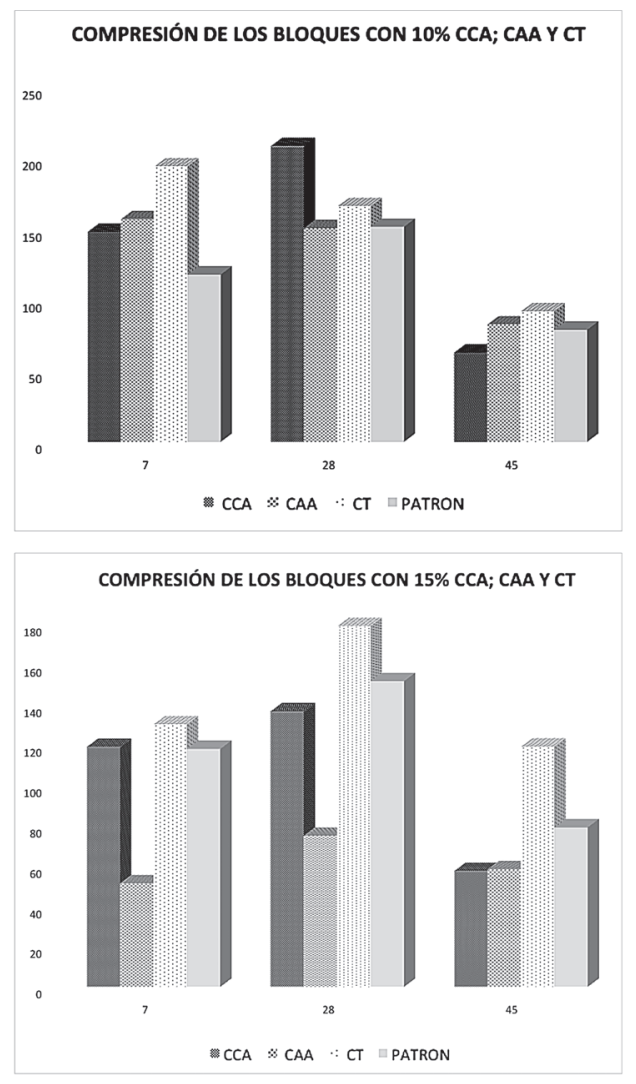

COMPRESIÓN DE LOS BLOQUES CON 20\% CCA; CAA Y CT

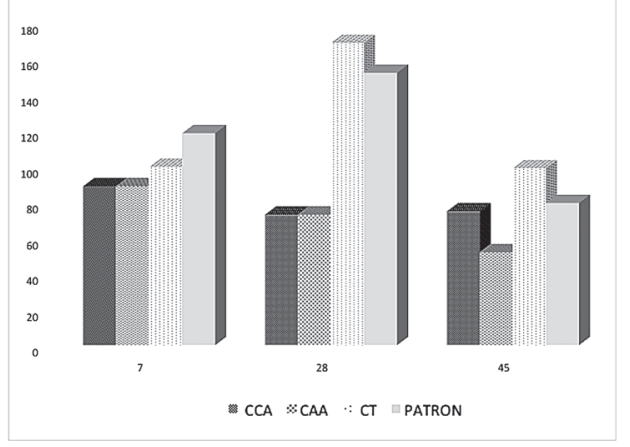

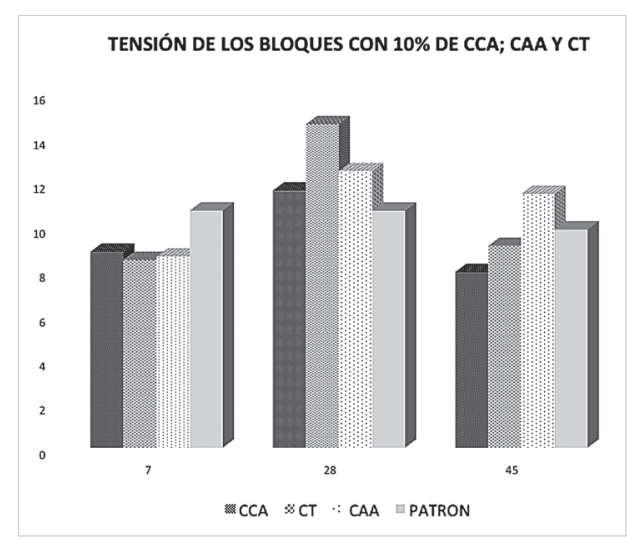

TENSIÓN DE LOS BLOQUES CON 15\% DE CCA; CAA Y CT

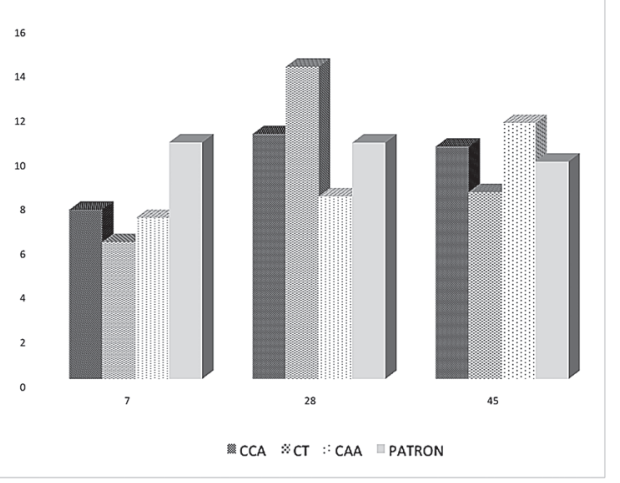

TENSIÓN DE LOS BLOQUES CON 20\% DE CCA;CAA Y CT

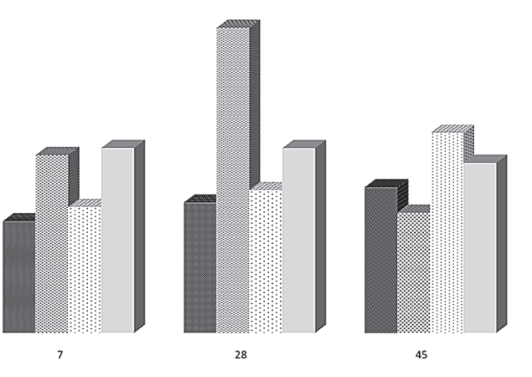

$\because C C A * C T \because C A A \square$ PATRON

Figura 3. Análisis comparativo de la resistencia a tensión y resistencia a la compresión de las adiciones por porcentaje en sus diferentes días de curado.

En la mayoría de los casos, la resistencia a la compresión y tensión de los bloques elaborados presenta una reducción a los 45 días de fraguado; esto significa que el efecto de hidratación es importante en la elaboración de los bloques y que los agregados residuales 
se ven afectados de forma negativa en la generación de resistencias mecánicas, donde aparentemente la adherencia de los materiales residuales y el cemento no fue demasiado buena, lo que ocasionó una disminución en la resistencia mecánica a la compresión y tensión cuando se aumentó a 45 días el fraguado de los bloques no estructurales.

De lo anterior se plantea que, en términos generales, los bloques con 28 días de curado presentan una mayor resistencia a la tensión en comparación con los demás días, teniendo como referencia el patrón, excepto los bloques que contienen como material residual la cascarilla de arroz, a la cual se recomienda moler para mejorar su efectividad.

\section{CONCLUSIONES}

Se lograron obtener bloques de concreto no estructurado con densidades promedio de $1,30 \mathrm{~g} / \mathrm{cm} 3$, que presentan resistencias mecánicas que varían entre 0.358 y 1.439 $\mathrm{MPa}$, las cuales presentaron características mecánicas similares a las comerciales, resultando interesantes para ser empleados en componentes de construcción liviana y de uso no portante, toda vez que no cumplen con la NTC 4076.

Los resultados obtenidos en los ensayos realizados permiten demostrar la viabilidad de utilizar los materiales cenizas de termoeléctrica y cenizas de cascarilla de arroz como aglomerante puzolánico desde el punto de vista mecánico, para sustituir parcialmente el cemento Portland, al presentarse valores cercanos y mayores en la mayoría de los casos en relación con el bloque patrón, a pesar de no cumplir con las especificaciones técnicas de la norma en ninguno de los casos analizados.
Los bloques ecológicos presentan un mejor comportamiento con respecto a la resistencia a la compresión y a la tensión cuando tienen 28 días de curado. Los porcentajes óptimos para la elaboración de los bloques ecológicos son aquellos que contenían el $15 \%$ de cenizas de termoeléctrica, lo cual es atribuido al tamaño de las partículas, como se muestra en el análisis granulométrico realizado.

Los bloques ecológicos diseñados con cenizas industriales de las centrales térmicas para porcentajes tanto del 10,15 y $20 \%$ siempre estuvieron por encima del patrón en lo que respecta a resistencia a la compresión, lo que indica que es factible reutilizar estos materiales para la elaboración de bloques de concreto no estructurado, gracias a que alcanza valores de resistencia similares al patrón que otorgan solución a los problemas ambientales generados por estos residuos.

\section{BIBLIOGRAFÍA}

[1]. Vásquez, R. y Bach Vigil, P. (2000). Las cenizas de cáscara de arroz; adición puzolánica en cemento y concreto. Universidad de Piura. Piura, Perú.

[2]. Hendriks, C. A. et al. (1998). Emission reduction of greenhouse gases from the cement industry. Fourth International Conference on Greenhouse Gas Control Technologies. Interlaken, August 30-September 2, 1998.

[3]. Vanderley, M. J. (2002). On the sustainability of the Concrete. Extended version of the paper commissioned by UNEP, Journal Industry and Environment.

[4]. Méndez, R. et al. (2010). Optimización 
de mezclas cal-puzolana destinadas a la construcción de materiales prefabricados no convencionales. II Simposio Aprovechamiento de residuos agroindustriales como fuente sostenible de materiales de construcción, Valencia.

[5]. Payá, J. et al. (2003). Evaluation of the pozzolanic activity of fluid catalytic cracking residue(FCC). Thermogravimetric analysis studies on FCC-Portland cement pastes. Cement Concrete Research 2003; 33:603-9.

[6]. Martirena, J. F. (2004). Una alternativa ambientalmente compatible para disminuir el consumo de aglomerantes de clinker de cemento Portland: el aglomerante cal-puzolana como adición mineral activa. Tesis doctoral, Universidad Central Marta Abreu de las Villas.

[7]. Ordóñez, L. (2007). Reutilización de la ceniza de cáscara de arroz como material deconstrucción: valoración yoptimización de sus propiedades puzolánicas. Tesis doctoral. Valencia: Departamento de Ingeniería de la Construcción y de Proyectos de Ingeniería Civil. Universidad Politécnica de Valencia.

[8]. Treviño, B., Gómez, I. (2002). Obtención de fases del cemento utilizando deshechos agrícolas e industriales, Universidad Autónoma de Nuevo León, Monterrey, México. Vol. V, No. 2, pp. 190-196.

[9]. Federación Nacional de Arroceros (Fedearroz) (2011). Estadísticas arroceras; bondades y beneficios del arroz, Bogotá, D.C.

[10]. Mattey, Pedro E., Robayo, Rafael A., Díaz, Jherson E., Delvasto, Silvio Arjona, Monzó,
José. (2015). Influencia del mezclado en dos etapas en la fabricación de ladrillos de mampostería con ceniza de cascarilla de arroz como agregado fino. Revista Colombiana de Materiales No. 5, pp. 242249.

[11]. Serrano, Tomás, Borrachero, M. Victoria, Monzó, José M. y Payà, Jordi. (2012). Lightweight mortars with rice husk: mix design and properties evaluation. Dyna, año 79, No. 175, pp. 128-136, Medellín.

[12]. Zain, M.F.M., Islam, M.N., Mahmud, F. y Jamil, M. (2011). "Production of rice husk ash for use in concrete as a supplementary cementitious material". Construction and Building Materials 25, pp. 798-805.

[13]. Chopra, D., Siddique, R. y Kunal. (2015). "Strength, permeability and microstructure of self-compacting concrete containing rice husk ash". Biosystems Engineering I, 30, pp. 72-80.

[14]. Maroto V., M. E. (1999). Environmental benefits of producing adsorbents materials from unburned carbon. The Pennsylvania State University. The Energy Institute and department of energy and geo-environmental engineering.

[15]. Centro de Investigaciones del Carbón. (1996). Universidad Nacional de Colombia, Centro de Investigaciones del Carbón, Bogotá.

[16]. Quijano, J. C. (2010). Análisis del comportamiento mecánico del cemento hidráulico, adicionados con cenizas volantes provenientes de la Central de Generación Térmica de Termopaipa. 
Trabajo de grado, Universidad Industrial de Santander, Santander, Bucaramanga.

[17]. Susan, A., Bernal, E. (2011). Mechanical and thermal characterisation of geopolymers based on silicate-activated metakaolin/slag blends. Journal of Materials Science, 46, 5477-5486.

[18]. Bouzoubaâ, M. Z. (2001). Mechanical properties and durability of concrete made with high-volume fly ash blended cements using a coarse fly ash Original Research. Cement and Concrete Research, 31 Issue 10, 1393-1402.

[19]. Chur Pérez. G. (2010). Evaluación del uso de la cascarilla de arroz como adición orgánica en bloques ecológicos de mampostería. Universidad de San Carlos, Guatemala.

[20]. Bizzotto, M., Natalini, M. y Gómez, Gaspar. (1998). Minihormigones con cascarilla de arroz natural y tratado como adición granular. Primer Congreso Internacional de Tecnología del Hormigón. Universidad Nacional del Nordeste. Buenos Aires, Argentina.

[21]. González Cuervo, C., Montaño Angarita, Á y Castro Rodríguez, D. (2011). Obtención y caracterización de geopolímeros; sintetizados a partir de ceniza volante y piedra pómez; utilizados para el desarrollo y mejoramiento del concreto. Universidad Industrial de Santander. Bucaramanga, Colombia.

[22]. Gómez Gonzales, A. (2009). Caracterización y utilización de puzolanas como aditivos minerales activos en cementos. Aplicación en viviendas de bajo coste. Universidad Politécnica de Valencia. Valencia, España.

[23]. Águila, I. y Sosa, M. (2008). Evaluación físico-química de cenizas de cascarilla de arroz; bagazo de caña y hoja de maíz y su influencia en mezclas de mortero; como materiales puzolánicos. Revista de la Facultad de Ingeniería U.C.V. Vol. 23. $\mathrm{N}^{\circ} 4$, Universidad Central, Venezuela, pp. 55-66.

[24]. Cabo Laguna, M. (2011). Ladrillo ecológico como material sostenible para la construcción. Universidad Pública de Navarra. España.

[25]. Ospina, M., Monzó, J., Payá, J., Borrachero, M., Mejía, R. y Delvasto, S. (2008). Utilización del residuo obtenido en el proceso de combustión de la cascarilla de arroz para la preparación de materiales de construcción de bajo coste con base cementicia. I Simposio Iberoamericano de Ingeniería de Residuos. Universidad Politécnica de Valencia. Valencia, España.

[26]. Batic, O., Giaccio, G., Zerbino, R. e Isaia, G. (2010). Las cenizas de cáscara de arroz y la reacción álcali sílice. VI congreso internacional sobre patologías y recuperación de estructuras. Argentina.

[27]. Aprile, A., Beecher, A., Bertoni, M., Bretti, J. y Eglis, I. (1999). Reutilización de cenizas de centrales térmicas. www. ingenieroambiental.com.ar. Argentina.

[28]. Sierra Aguilar, J. (2009). Alternativas de aprovechamiento de la cascarilla de arroz en Colombia. Universidad de Sucre. Sincelejo, Colombia. 
[29]. Salazar, A. (2005). Experiencia de reciclaje en la producción de materiales de construcción. Quinto congreso nacional disposición final de residuos sólidos. Universidad del Cauca. Cali, Colombia.

[30]. Álvarez Alonso, M. y Río Suárez, O. (1991). Elementos prefabricados de hormigón con cenizas centrales térmicas. Materiales de construcción. Vol. 41. No. 224. España.

[31]. Lamb Bernal, C. y Ramírez Carmona, M. (2008). Elaboración industrial de bloques de concreto empleando ceniza volante. Revista Investigaciones Aplicadas. No. 4, Universidad Pontificia Bolivariana. Medellín, Colombia, p. 8.

[32]. Santaella Valencia, L. y Salamanca Correa, R. (2004). Comportamiento del concreto con bajos porcentajes de ceniza volante (Termopaipa IV) y agua constante. Ciencia e Ingeniería Neogranadina ISSN (versión impresa): 0124-8170. No. 14. Universidad Militar Nueva Granada, Bogotá, Colombia, pp. 1-7.

[33]. Molina, Moragues Terrades y Gálvez Ruiz. (2008). La influencia de las cenizas centrales térmicas como sustituto parcial del cemento Portland en la durabilidad del hormigón: propiedades físicas; difusión del ión cloruro y del dióxido de carbono". Anales de Mecánica de la Fractura 25. Vol. 2. Universidad Politécnica de Madrid. Madrid, España.

[34]. Burgos, D., Angulo, D. y Mejía de Gutiérrez, R. (2011). Durabilidad de morteros adicionados con cenizas centrales térmicas de alto contenido de carbón. Rev. LatinAm. Metal. Mat. Universidad del Valle. Cali, Colombia, pp. 61-70.
[35]. Hernández Toledo, U., Alavés Ramírez, R. y Montes García, P. (2009). Ceniza de coco y hoja de pino para su uso como puzolana. Centro Interdisciplinario de Investigación para el Desarrollo Integral Regional I.P.N. Unidad Oaxaca. Oaxaca, México.

[36]. Zamorano, M. (2013). Hormigón barato con cenizas de la combustión de adición de olivo.

[37]. Escalera Cruz, Alejandro, Payá Bernabeu, Jordi, Borrachero Rosado, María Victoria, Soriano Martínez, Lourdes y Monzó Balbuena, José María. (2012). Estudio de morteros de cemento Portland con ceniza de rastrojo de maíz: posibilidades de uso en construcciones rurales. Instituto de Ciencia y Tecnología del Hormigón. Universitat Politécnica de Valencia, España.

[38]. Quiceno Villada, D. y Mosquera Gutiérrez, M. (2010). Alternativas tecnológicas para el uso de la cascarilla de arroz como combustible. Universidad Autónoma de Occidente. Cali, Colombia.

[39]. Pozas del Río, J. (2008). Contribución al empleo en Cuba de la ceniza de cáscara de arroz obtenida en horno artesanal; como una adición activa en morteros estructurales de base cemento". Instituto Superior Politécnico José Antonio Echeverría. Habana, Cuba.

[40]. Suárez Silgado, S. (2010). Mezclas binarias y ternarias basadas en cenizas centrales térmicas. Influencia del activador sobre la formación de fases y resistencias mecánicas. Universidad Politécnica de Catalunya. Barcelona, España. 